\title{
LA UNIVERSIDAD COMO ESPACIO DE EMPODERAMIENTO. \\ LOS SEMINARIOS DE ORATORIA Y DEBATE, \\ UNA OPORTUNIDAD PARA EL FOMENTO DEL LIDERAZGO FEMENINO
}

THE UNIVERSITY AS A SPACE OF EMPOWERMENT. THE SEMINARS OF ORATORY AND

DEBATE, AN OPPORTUNITY FOR THE PROMOTION OF FEMALE LEADERSHIP

\author{
María Amparo Calabuig Puig \\ Universidad Miguel Hernández de Elche
}

RESUMEN

El presente trabajo pretende plasmar la implementación de una experiencia piloto en materia de oratoria y debate llevada a cabo en la universidad Miguel Hernández de Elche, planteándose dicho proyecto desde los valores de la igualdad efectiva de mujeres y hombres. Y es que la Universidad se presenta como una esfera privilegiada desde la que debemos proyectar los valores del empoderamiento en igualdad de condiciones al resto de la sociedad, teniendo dicha institución educativa una especial responsabilidad como agente socializador, trasmisor de valores y referente del pensamiento crítico.

Palabras clave: universidad, empoderamiento, liderazgo, igualdad efectiva, oratoria y debate.

\section{ABSTRACT}

The present work aims to reflect the implementation of a pilot experience in the area of speech and debate, carried out at the Miguel Hernández University of Elche. This project is based on the values of effective gender equality between women and men. After all, the University presents itself as a privileged sphere from which we must project the values of empowerment in equal conditions to the rest of society, having a special responsibility as socializing agent, transmitter of values and referent of critical thinking. Keywords: University, Empowerment, Leadership, Gender Equality, Speech and Debate. 


\section{Introducción}

A pesar de los incuestionables avances sociales, culturales y legales, aún perviven -de un modo más o menos evidente- determinados estereotipos y roles tradicionales, intensamente conectados con la perenne infravaloración de la actividad femenina. Muestra de ello es la segregación horizontal y vertical del trabajo, los diferentes usos del tiempo, la asimetría entre la maternidad y la paternidad, la consecuente brecha salarial, la discriminación en materia de cotización o pensiones y un largo etcétera. Así lo reflejan los datos que arroja el informe Mujeres y hombres en España (2015) del Instituto Nacional de Estadística de España, pues, entre otras cifras de interés, indica que el $91,9 \%$ de las mujeres -de 10 años o más- realizan tareas domésticas y se ocupan del cuidado de personas dependientes durante 4 horas y 29 minutos diarios, frente al $74,7 \%$ de los hombres que dedican en promedio 2 horas y 32 minutos (INE, 2015: 384). Fenómenos intensamente conectados que, sin embargo, aún no son tan evidentes para el alumnado inmerso en el «oasis universitario», pero que sin lugar a dudas han limitado y limitan en la práctica las vidas y por ende las carreras profesionales de las mujeres, coartando en definitiva sus posibilidades para ejercitar de forma efectiva, eso es plenamente y en igualdad con los varones, los derechos de los que ambos son titulares (Collado, 2014: 186).

En este sentido, si hay un ámbito en el que aún se hace más patente la permanente desigualdad de género es el del poder ${ }^{1}$-entendido y ejercicio mayoritariamente desde la construcción de lo masculino-. Los puestos de dirección y de toma de decisiones han estado tradicionalmente $-y$ están-, vinculados mayoritariamente a los hombres. Y si bien parece que la participación de las mujeres avanza progresivamente, en realidad continúa presentando resultados aún peores que los correspondientes al mercado laboral en su globalidad (Figueruelo, 2014: 243). Situación que encuentra sus raíces en la construcción social patriarcal.

En relación a lo expuesto los datos no dejan lugar a dudas, según el informe del INE citado anteriormente, en el año 2015, el porcentaje de mujeres en el conjunto de Consejos de Administración de las empresas que forman parte del lbex-35 era del 19,4\%. Si se considera el cargo ejercido en los consejos de administración, los porcentajes de mujeres entre quienes ostentaban la presidencia -5,7\%- o vicepresidencia -2,4\%- eran aún más bajos. En el año 2015 las mujeres representaban algo menos de la tercera parte del total de quienes ocupaban los órganos superiores y los altos cargos de la Administración General del Estado.

1 Max Weber en su obra Economía y sociedad, esbozo de la sociología comprensiva (1922), define el poder como la probabilidad de que un actor dentro de un sistema social esté en posición de realizar su propio deseo, a pesar de las resistencias. 
En el ámbito de la representación política, tras las elecciones generales del 26 de junio de 2016, en el Congreso de los Diputados, el porcentaje de mujeres electas fue del $39,4 \%$. Y en agosto de 2016, el porcentaje de mujeres en el conjunto de cargos ejecutivos de los partidos políticos se sitúa en un $37,5 \%$. Pudiendo observar un lógico aumento de la presencia de mujeres en aquellos ámbitos en los que se aplica, obligatoriamente, el sistema de «cuotas» de la Ley 3/2007, de 22 de marzo, para la igualdad efectiva de mujeres y hombres ${ }^{2}$ y un estancamiento en aquellos en los que la misma norma optó por la mera recomendación -soft law-, un claro ejemplo de ello son los consejos de administración de las empresas $^{3}$.

Antes de continuar cabe poner la lupa en el ámbito que nos ocupa, la universidad, que a pesar de lo deseable no escapa a dicha situación. Pues en su propio seno se da el denominado «efecto tijera», es decir, a pesar de contar con una mayoría de alumnas $-54,9 \%$ - su presencia va descendiendo considerablemente a medida que asciende la escala universitaria, encontrando en el curso 2014/2015 un 20,8\% de catedráticas en el conjunto del Estado español (Pastor y Acosta, 2016: 252). Por no hablar de su baja presencia como decanas de facultades y escuelas, y ya anecdótica como rectoras.

Al respecto, cabe destacar que el fenómeno de la baja presencia de mujeres en el vértice de las organizaciones, y de los elementos que interactúan para perpetuarlo a pesar de estar presentes en las bases y puestos intermedios, ha sido acuñado de diferentes modos a lo largo del tiempo. A partir de los años ochenta del término «techo de cristal» -glass ceiling-, haciendo una alusión metafórica a «las barreras invisibles que impiden a muchas mujeres con elevada cualificación y capacidad personal y profesional acceder a los niveles de dirección y responsabilidad más elevados y promocionar en ello» (SegermanPeck, 1991: 243). Estas barreras invisibles son obstáculos implícitos derivados de prejuicios psicológicos y organizativos (Ramos, 2005: 47). Posteriormente se consideró más apropiado el término «laberinto de cristal» (Barberá, Ramos y Candela, 2011 : 174). Esta metáfora trata

\footnotetext{
2 A través de la Disposición Adicional $1^{a}$ de la Ley de lgualdad se modificaron diferentes preceptos de la Ley 5/1985, de 19 de junio, del Régimen Electoral General, destacando la nueva redacción del artículo 44 bis, relativo a la composición equilibrada de mujeres y hombres en las elecciones al Congreso, al Senado, a los ayuntamientos, al Parlamento Europeo y a los parlamentos autonómicos, de tal modo que, en el conjunto de la lista, los candidatos de cada uno de los sexos supongan como mínimo el 40 por 100 y no más del 60 por 100. Además esta proporción mínima de cada uno de los sexos deberá mantenerse en cada tramo de cinco puestos de la lista electoral en el caso de que el tramo de la lista no alcance el número de cinco puestos, o que el número de puestos a cubrir sea inferior a cinco, la proporción de hombres y mujeres será lo más cercana posible al equilibrio numérico En todo caso, deberá mantenerse la proporción exigible en el conjunto de la lista. Asimismo deberá aplicarse la misma regla numérica a las listas de suplentes.

3 Artículo 75 de la Ley de lgualdad: «Participación de las mujeres en los Consejos de administración de las sociedades mercantiles: 1. Las sociedades obligadas a presentar cuenta de pérdidas o ganancias no abreviada procurarán incluir en su Consejo de Administración un número de mujeres que permita alcanzar una presencia equilibrada de mujeres y hombres en un plazo de ocho años a partir de la entrada en vigor de esta Ley».
} 
de explicar que no existen barreras imposibles de atravesar para llegar a la cúspide y que, además, éstas no son totalmente invisibles. Las mujeres no tienen denegado el acceso a los puestos más altos, sino que, a diferencia de los hombres, deben sortear una mayor cantidad de obstáculos para poder llegar a ellos. En definitiva, responde a la idea de que para ellas los caminos correctos para poder avanzar resultan más difíciles de hallar (Lupano, 2009: 73). Desde las ciencias sociales se han desarrollado diferentes hipótesis explicativas sobre los elementos que conforman dicho fenómeno, coincidiendo la mayoría en la interrelación de factores externos a las mujeres -estructura y cultura organizacional y los estereotipos de género-, internos -rasgos motivacionales y de personalidad-, y explicativos interactivos -responsabilidades familiares y promoción profesional- (Ramos, 2005: 39).

En consecuencia, y atendiendo al trascendente impacto social que tiene, la universidad no puede obviar dicha problemática, que va mucho más allá de la cantidad de egresadas y egresados, o de sus correspondientes calificaciones, comprometiéndose como formadora de las personas que serán el motor de la transformación. Avanzando del mero cumplimiento de la letra, bajo mínimos en la mayoría de ocasiones, de la Ley de lgualdad, y de la Ley Orgánica de Universidades $6 / 2001^{4}$, a la consecución de su espíritu. Y en definitiva, en aras del cumplimiento del principio de igualdad de oportunidades y no discriminación -artículo 14- reconocido en nuestra Carta Magna y del mandato que la misma hace a los poderes públicos para que pongan los medios necesarios en vistas de garantizar el goce efectivo de los derechos en condiciones de igualdad-artículo 9.25. Por no olvidar el artículo 35.16 que garantiza el derecho a la promoción en el trabajo y prohíbe la discriminación laboral por razón de sexo.

Para ello, como punto de partida deberá incorporar la perspectiva de género de un modo transversal, teniendo siempre presente la existencia del problema y lo más importante, de dónde viene. Y luego, implementar medidas concretas para la deconstrucción

\footnotetext{
4 La Ley de Universidades contiene reiterados mandatos relativos al fomento de la enseñanza y la investigación sobre el significado y alcance de la igualdad entre mujeres y hombres, la inclusión en los planes de estudio de enseñanzas, la creación de postgrados específicos y la realización de estudios e investigaciones especializadas en dicha materia.

5 Artículo 9.2 de la Constitución Española: «Corresponde a los poderes públicos promover las condiciones para que la libertad y la igualdad del individuo y de los grupos en que se integra sean reales y efectivas; remover los obstáculos que impidan o dificulten su plenitud y facilitar la participación de todos los ciudadanos en la vida política, económica, cultural y social». Y en definitiva con el objetivo de dar efectividad al artículo 14 de la Carta Magna: «Los españoles son iguales ante la ley, sin que pueda prevalecer discriminación alguna por razón de nacimiento, raza, sexo, religión, opinión o cualquier otra condición o circunstancia personal o social».

6 Artículo 35.1 de la Constitución Española: «Todos los españoles tienen el deber de trabajar y el derecho al trabajo, a la libre elección de profesión u oficio, a la promoción a través del trabajo y a una remuneración suficiente para satisfacer sus necesidades y las de su familia, sin que en ningún caso pueda hacerse discriminación por razón de sexo».
} 
de los roles tradicionales, el fomento en las aulas del empoderamiento en igualdad ${ }^{7}$ y el desarrollo de liderazgos transformacionales ${ }^{8}$. En ese sentido los seminarios de "oratoria y debate» se nos presentan como una oportunidad para el fomento del liderazgo femenino y la deconstrucción de los estereotipos que lo limitan -por ser un entorno encaminado a la toma de la palabra, a la gestión de decisiones, a la dirección de grupos, etc. Además de apostar por un trabajo en equipo y un liderazgo transformacional ${ }^{9}$ (Ramos, 2011 : 79-88), que supere las clásicas rémoras. La presente propuesta consiste en un relato de la implementación de una experiencia piloto y la correspondiente evaluación de sus resultados, pudiendo servir, no solo de reflexión, sino también de ejemplo o inspiración para nuevas dinámicas en otros centros.

Por ende, la universidad se nos presenta como esfera privilegiada desde la cual debemos proyectar los valores del empoderamiento en igualdad de condiciones al resto de la sociedad. Teniendo la misma una especial responsabilidad como agente socializador, trasmisor de valores y referente del pensamiento crítico.

\section{La experiencia del Taller Permanente de Oratoria y Debate en la Universidad Miguel Hernández de Elche (TOD UMH)}

La Facultad de Ciencias Sociales y Jurídicas de Orihuela y el Departamento de Ciencia Jurídica de la Universidad Miguel Hernández ${ }^{10}$ han puesto en marcha durante el curso académico 2015/2016 y el presente 2016/2017 el Taller Permanente de Oratoria y Debate (TOD) $\mathrm{UMH}^{11}$. Un proyecto que ha buscado ofrecer formación continuada y de calidad en materia de oratoria y debate al alumnado de dicha universidad.

\footnotetext{
7 El término empoderamiento referido al aumento de la participación de las mujeres en el acceso al control de recursos materiales, intelectuales e ideológicos hasta llegar a una contribución plena para la construcción de la sociedad, permitiendo su participación en igualdad de condiciones a todos los niveles. Ello supone para las mujeres ganancias en autoestima, educación, información y derechos: y por lo tanto, poder. En los últimos tiempos este término ha ido adquiriendo un nuevo sentido, entendiendo que hace también referencia a la toma de conciencia del poder que, de forma individual y colectiva, ostentan las mujeres, y que tiene que ver con la recuperación de su propia dignidad como personas. En definitiva, la dignificación, la autonomía, para que puedan sentirse dueñas de sus decisiones, y vivir en paz, justicia e igualdad. En este sentido atendemos a la conceptualización y desarrollo de estrategias planteadas por la Organización de Naciones Unidas, a través, principalmente de sus Conferencias Mundiales.

8 Orientado a la innovación, el trabajo cooperativo en equipo, la puesta en valor de las potencialidades de todas las personas que componen el grupo y el aprovechamiento de todos los recursos disponibles.

9 Liderazgo basado en las relaciones horizontales, superando las verticales de subordinación; la implicación efectiva de todos los miembros del equipo y el reconocimiento de su potencial, fomentando el desarrollo personal y colectivo; la motivación orientada a objetivos comunes y las relaciones basadas en el respecto.

10 En adelante UMH.

11 En adelante TOD UMH.
} 
Su primera puesta en práctica, llevada a cabo en el curso 2015/2016,12 contó con una excelente acogida entre el alumnado de titulaciones como Ciencias Políticas y Gestión Pública, Derecho, Periodismo, Dirección y Administración de Empresas e incluso programas de doctorado. Un nutrido grupo de alumnas y alumnos que trabajaron, tanto de forma individual como en equipo, en talleres que abordaron el desarrollo de discursos, argumentación, búsqueda de información, uso de fuentes, proyección de la voz, comunicación no verbal, miedo escénico, etc. Cuestiones todas de gran trascendencia para el desarrollo personal y profesional, que también fueron puestas en práctica a través de una liga interna -Liga de Debate TOD UMH-y otra externa -Lliga de Debat Universitària de la Xarxa Vives-.

Pero si algo ha caracterizado al TOD UMH ha sido la aplicación de la perspectiva de género durante todo el proceso de planteamiento e implementación, es decir, de un modo transversal.

La incorporación de la perspectiva de género como herramienta conceptual o metodológica ha permitido centrar la intervención en la construcción social del sexo (el género) y, por tanto, señalar aquellos aspectos ideológicos o culturales $y$, por tanto, reinterpretables y mutables que explican o son el origen de la situación de vulnerabilidad (Nuño, 2014: 393).

Primero en la elaboración del proyecto, luego como criterio básico e imprescindible en el personal docente, en las formaciones, materiales, cartelería, publicidad (...). En definitiva, como elemento presente tanto en todas y cada una de las sesiones de formación como a través del desarrollo de módulos específicos sobre: lenguaje inclusivo, conceptos básicos en materia de igualdad, diferentes usos del tiempo de mujeres y hombres, Violencia de Género (...).

A continuación, realizaremos un análisis y evaluación de las medidas llevadas a cabo desde la perspectiva de género -en base a la programación ya cerrada, correspondiente al curso 2015/2016.

\subsection{Programación del curso TOD UMH (2015/2016)}

- Taller I: Formación básica en oratoria y debate, 12 y 19 de diciembre de 2015, 16 horas).

- Taller II: El arte de comunicar, 20 y 27 de febrero de 2016 (8 horas). 
- Programa de debate Derribando Muros en la Radio UMH sobre la «brecha salarial» ${ }^{13}$, 7 de marzo de 2016. Programa de 1h. Participación de alumnado del TOD UMH.

- Taller III: La elaboración de un BUEN DISCURSO, 12 de marzo de 2016 (4 horas).

- Taller IV: Sexo, Género...Hoy, en el marco del TOD UMH. Seminario teóricopráctico sobre conceptos básicos en materia de igualdad efectiva -sexo, género, roles, estereotipos-. Especialmente aplicado a los roles que jugamos en una clase, en una exposición o en un debate -tanto en su preparación como en la puesta en práctica-. 13 de abril de 2016 (4 horas).

- Taller V: El acceso de la mujer a la participación política. Debate a raíz del visionado de la película «Clara Campoamor, la mujer olvidada»,8 marzo de 2016 (4horas).

- Taller VI: Formación en lenguaje inclusivo y no estereotipado La importancia de tratarse bien en el discurso, 17 marzo de2016 (4horas).

Al finalizar el taller, el equipo de dirección del TOD UMH, representantes de la Unidad de Igualdad y del Centro de Investigación de Estudios de Género (CIEG) de la universidad, así como del Ayuntamiento de Orihuela inauguraron la muestra ${ }_{j}$ No tiene nombre! Lenguaje para la lgualdad. La exposición de la artista Diana Raznovich, compuesta por 30 carteles relacionados con el lenguaje inclusivo, se enmarcó dentro de las actividades que la UMH organizó para conmemorar el Día Internacional de la Mujer. La muestra se pudo visitar en el vestíbulo de la Facultad de Ciencias Sociales y Jurídicas de Orihuela.

- Taller VII: Cómo convertir tus debilidades en fortalezas, 9 de abril de 2016 (4 horas).

- Liga de debate TOD UMH'14. La primera fase se llevó a cabo el 12 de abril(5horas) y la final el 14 de abril 2016 (2horas).

- Participación en la XII Lliga de Debat Universitària de la Xarxa Vives, celebrada en la Universitat Politècnica de Valènica del 25 al 29 de abril de 2016.Los criterios de selección, preparación y funcionamiento del equipo representante de la UMH en dicha liga de debate fueron los correspondientes al TOD UMH. Estando compuesto el mismo por 4 alumnas y 4 alumnos del Grado en Ciencias Políticas y Gestión Pública ${ }^{15}$.

\footnotetext{
13 Enlace del programa de radio: http://radio.umh.es/2016/03/07/debate-sobre-el-dia-por-la-igualdad-salarial-derribando-muros7-de-marzo-de-2016/

14 Los vídeos de los debates están disponible en elcanalYOUTUBEUMH,enlalistadereproducción TOD UMH:hHtps://www.youtube. com/playlist?list=PLCIKgnzRFYe7GNtaPeGl5-XTLtepzz7w-

15 Más información sobre dicha liga en:http://www.vives.org/serveis/lliga-de-debat/Iliga-de-debat-universitari/lliga-de-debat-universitaria-2016/
} 


\subsection{Participación del alumnado y profesorado}

En todos los talleres se aplicó como criterio de admisión la presencia/participación equilibrada de alumnas y alumnos ${ }^{16}$, atendiendo en base a ello al orden de inscripción. Del mismo modo se aplicó dicho criterio en lo relativo al profesorado y al jurado de las ligas.

En este punto se hace imprescindible aclarar el concepto presencia/participación equilibrada por ser uno de los ejes fundamentales de la presente experiencia. Desde el TOD UMH hemos considerado imprescindible hacer hincapié en la diferencia entre la presencia -cuantitativay la participación -cualitativa- equilibrada considerando ambos requisitos indispensablemente complementarios. Pues en demasiadas ocasiones se tiende a cumplir con el requisito numérico dejando al lado la participación efectiva. Siendo al fin y al cabo la combinación de los dos aspectos, el cuantitativo y el cualitativo, el camino para el empoderamiento femenino, orientado al aumento de la participación de las mujeres en el acceso al control efectivo de recursos materiales, intelectuales e ideológicos permitiendo su participación en igualdad de condiciones a todos los niveles. Pretensión, la de la presencia/participación equilibrada sustantiva, que sin lugar a dudas aporta beneficios, tanto individuales como colectivos, no solo al alumnado universitario, sino a la sociedad en su conjunto, además de ser una exigencia inaplazable, en términos de justicia social y de puesta en valor del conjunto de los recursos humanos.

Finalmente, los datos expuestos a continuación, basados en el criterio meramente cuantitativo, pretenden ofrecer una visión nítida de cómo se ha cumplido con la presencia equilibrada. Sim embargo, en base a lo expuesto, no podemos dejar de entenderlos desde la óptica de la participación efectiva de hombres y mujeres, pues así lo ha intentado garantizar la organización en todo momento:

\begin{tabular}{|l|c|c|c|}
\hline ASISTENCIA & TOTAL & ALUMNOS & ALUMNAS \\
\hline TALLER I & 46 & $52 \%$ & $48 \%$ \\
\hline TALLER II & 43 & $51 \%$ & $49 \%$ \\
\hline PROGRAMA DE RADIO & 4 & $50 \%$ & $50 \%$ \\
\hline TALLER III & 25 & $64 \%$ & $36 \%$ \\
\hline TALLER IV & 35 & $54 \%$ & $46 \%$ \\
\hline TALLER V & 45 & $44 \%$ & $56 \%$ \\
\hline TALLER VI & 29 & $55 \%$ & $45 \%$ \\
\hline TALLER VII & 22 & $59 \%$ & $41 \%$ \\
\hline LIGA DE DEBATE TOD UMH & 34 & $53 \%$ & $47 \%$ \\
\hline LIGA DE DEBATE XARXA VIVES & 8 & $50 \%$ & $50 \%$ \\
\hline
\end{tabular}




\begin{tabular}{|l|c|c|}
\hline PROFESORADO & PROFESOR & PROFESORA \\
\hline TALLER I & 1 & 1 \\
\hline TALLER II & 1 & 1 \\
\hline PROGRAMA DE RADIO & 0 & 1 \\
\hline TALLER III & 1 & 0 \\
\hline TALLER IV & 1 & 1 \\
\hline TALLER V & 1 & 1 \\
\hline TALLER VI & 0 & 1 \\
\hline TALLER VII & 1 & 1 \\
\hline LIGA DE DEBATE TOD UMH & 7 & 7 \\
\hline TOTALES ${ }^{7}$ & 13 & 14 \\
\hline
\end{tabular}

\section{Criterios y objetivos de actuación del TOD UMH desde la perspectiva de género}

Criterio 1: Identificación y supresión de contenidos sexistas visuales y escritos (ejemplos, imágenes...) en los materiales de los talleres de formación y publicidad.

a. Reunión con el profesorado.

b. Control de contenidos.

c. Cartelería con presencia de referentes femeninos y masculinos (Clara Campoamor, Enma Watson, Michelle Obama, Martin Luther King, Miguel Hernández y MalalaYousafzai).

d. Lenguaje inclusivo en toda comunicación con el alumnado.

e. Taller sobre conceptos básicos en materia de igualdad efectiva -sexo género, estereotipos, roles, etc.- para el alumnado.

f. Taller de especialización en lenguaje inclusivo e identificación de contenidos sexistas orientado al alumnado.

g. Incorporación como criterio de evaluación de los debates por parte del jurado.

Criterio 2: visibilización de ejemplos de empoderamiento femenino.

a. En cartelería.

b. En los ejemplos del contenido.

17 No se han incorporado los datos relativos al profesorado/jurado de la XII Lliga de Debat Universitària de la Xarxa Vives por no depender su gestión de la UMH. 
c. En la utilización de discursos de referencia.

d. Visionado de la película de Clara Campoamor.

Criterio 3: sensibilización.

a. Seminarios especializados sobre conceptos básicos y de identificación de elementos sexistas.

b. Programas de radio de debate sobre: brecha salarial, violencia de género e igualdad efectiva.

Criterio 4: exigencia de la presencia/participación equilibrada en los equipos, entre el profesorado, jurado y en la composición de los equipos formados por el alumnado.

a. Como requisito de admisión.

b. Exigencia en el reglamento, requisito obligatorio para optar a la participación en las ligas de debate.

c. En el desarrollo de cada sesión práctica.

Criterio 5: Redistribución de roles internos en los equipos.

a. Rotación de las funciones de: oradora/orador principal; refutador/refutadora y documentalistas.

Objetivo 1: Ofrecer un aprendizaje transversal basado en la comunicación inclusiva-tanto oral como visual-y a la vez dotar al alumnado de las herramientas necesarias para identificar contenidos sexistas.

Objetivo 2: Visibilizar entre el alumnado referentes femeninos en el ámbito del poder y la toma de decisiones.

Objetivo 3: Transmitir al alumnado los conceptos básicos en materia de género e igualdad, además de abordar los fenómenos que derivan de los mismos, haciéndoles conscientes del impacto que provoca en sus vidas y de cómo nos afecta de modo diferente a mujeres y a hombres.

Objetivo 4: Incorporar en el alumnado de los valores de participación/presencia equilibrada, atendiendo a las raíces y motivaciones de la misma. En consecuencia, hacerles capaces de identificar y experimentar del proceso de empoderamiento femenino que supone.

Objetivo 5: Fomentarla superación de los roles de género tradicionales desde las propias dinámicas de grupo en el aula e impulsar el liderazgo femenino y en definitiva transformacional.

Objetivo 6: Promover el trabajo cooperativo en equipo, la puesta en valor de las 
potencialidades de todas las personas que componen el grupo y el aprovechamiento de todos los recursos disponibles.

Objetivo 7: Concienciar sobre la necesidad del desarrollo efectivo de los anteriores objetivos y de la importancia de su proyección en el resto de ámbitos de la vida del alumnado. Además de visibilizar el beneficio que puede aportar en sus vidas dicho cambio de paradigma.

\section{Resultados, logros y retos}

I. Desde el punto de vista de la presencia/participación equilibrada la organización del TOD UMH se muestra satisfecha, pues generalmente se ha cumplido, tanto en la admisión del alumnado, como en la selección del profesorado, la configuración de los grupos de debate y jurados. Sin embargo, debemos seguir trabajando en este sentido, pues la tendencia mayoritaria ha sido a contar con un pequeño margen a favor de la cantidad de alumnos. De hecho, si atendemos a los datos de solicitud de matrícula, la cantidad de alumnos que a priori se han inscrito ha sido generalmente superior al de alumnas, debiendo esforzarse la organización por atraer en mayor medida a las alumnas.

II. EI TOD UMH ha logrado la participación de un nutrido grupo de alumnado, sin embargo, debe establecer mecanismos para su completa fidelización. Pues lo óptimo para lograr la formación integral es la asistencia a la mayoría de los talleres, y sin embargo en algún caso la asistencia ha sido intermitente.

III. En esta primera puesta en práctica hemos contado con alumnado, principalmente, de los Grados en Ciencias Políticas y Gestión Pública, Derecho y Administración y Dirección de Empresas. Sin embargo, resulta necesario ampliar el abanico de titulaciones implicadas en el TOD UMH. Por ser dicha formación de transversal de vital importancia para el alumnado de cualquier titulación.

IV. Todos los seminarios y talleres han tenido una potente carga práctica que ha recaído en el alumnado -individualmente y en grupo-, siempre tutelado por el profesorado. No obstante, resulta necesario un mayor grado, que durante esta primera experiencia ya ha sido alto, de dinámicas de trabajo en equipo. En todo caso las mismas deberán atender al criterio de rotación de roles por ejemplo la función de documentación recaiga siempre en uno de los dos sexos, así como el rol de orador/oradora -pasando de la mera presencia equilibrada a la idea de participación equilibrada efectiva-. El profesorado siempre deberá dar directrices de trabajo orientadas al liderazgo respetuoso, dialogante y constructivo.

V. A raíz de la insistencia durante las formaciones y correspondientes feedback con el alumnado, el jurado y la organización valora muy positivamente la ausencia de contenidos sexistas 
o actitudes irrespetuosas. No obstante, dicha exigencia debe de continuar muy presente en próximas programaciones.

Durante el curso académico 2016/201718se está desarrollando una nueva programación basada en la analizada en el presente trabajo, intentando incorporar las propuestas aquí planteadas.

En definitiva, la Universidad como poder público no solo tiene el deber de abanderar y respetar los valores de la igualdad en su seno, sino que tiene el compromiso social de hacerlo de forma proactiva, trabajando desde dentro hacia fuera, con el objetivo de transformar las estructuras sociales. Para ello, por una parte, cuenta con la autonomía que se le reconoce a su favor, puesto que le permite crear unas estructuras y una conciencia no predeterminada por el resto de poderes públicos. Y por otra cabe considerar su especial naturaleza, que le obliga a estar en constante contacto directo con la esfera privada. Por estos motivos, el ámbito universitario acumula todos los ingredientes para constituir un enclave estratégico a fin de proyectar principios los principios de la igualdad efectiva al resto de la sociedad.

Por ello, no solamente debemos formar superando los clásicos roles y estereotipos, haciéndoles capaces de identificarlos y de entender sus repercusiones -en sus vidas y en las de su entorno-; sino que también debemos esforzarnos en mejorar su empleabilidad en igualdad de condiciones, atendiendo a los condicionamientos previos que puedan traer consigo, y dándoles las herramientas para superarlos. Todo ello en base a un concepto de liderazgo que supere al clásico, cargado de los valores del diálogo, la cooperación y el respeto. De este modo contaremos con egresadas y egresados mejor formadas/os, pero además con profesionales que esperemos apliquen y trasladen dichos valores a sus entornos - o como mínimo tendrán las herramientas para hacerlo-. No obstante, esta experiencia no puede quedar aislada, sino que debe ir acompañada de toda una red de medidas y estrategias a desempeñar en la universidad, en sentido amplio. Es aquí donde radica el reto.

La sociedad reclama a la universidad del futuro una activa participación en sus procesos vitales. Por esta razón, la acción de la universidad no debe limitarse a la transmisión del saber; debe generar opinión, demostrar su compromiso con el progreso social y ser un ejemplo para su entorno.

La igualdad entre hombres y mujeres, los valores superiores de nuestra convivencia, el apoyo permanente a las personas con necesidades especiales, el fomento del valor del diálogo, de la paz y de la cooperación entre los pueblos, son valores que la universidad debe cuidar de manera especial (Preámbulo de la Ley Orgánica 4/2007, de 12 de abril, por la que se modifica la Ley Orgánica 6/2001, de 21 de diciembre, de Universidades). 


\section{Referencias bibliográficas}

BarberÁ, Esther, Ramos, María Amparo y Candela, Carlos (201 1): «Laberinto de cristal en el liderazgo de las mujeres», Psicothema. N'2, pp. 173-179.

Collado MateO, Concepción (2014): «Algunas notas para la perspectiva de género en la docencia de Derecho Constitucional en la toma de decisiones», en lgualdad y democracia: el género como categoría de análisis jurídico, València, Corts Valencianes, pp. 271-683.

Figueruelo BurRiezA, Ángela (2014): «lgualdad de género en la toma de decisiones, sobre la composición equilibrada de los consejos de administración de las grandes empresas», en Igualdad y democracia: el género como categoría de análisis jurídico, València, CortsValencianes, pp. 242-252.

LuPANO, María Laura (2009): «Nuevas metáforas acerca de las mujeres líderes», Psicodebate. N9, pp. 65-80.

NuÑo Gómez, Laura (2016): Evolución del tratamiento de la igualdad de género, en Deontología, principios jurídicos básicos e igualdad, Madrid, Tecnos, pp. 393-401.

Pastor Gonsalbez, Inma y Acosta Sarmiento, Ana (2016): «La institucionalización de las políticas de igualdad de género en la Universidad española. Avances y retos», Investigaciones Feministas. Vol. $7 \mathrm{~N}^{\circ} 2$, pp. 247-271.

Ramos, María Amparo (2005): Mujeres y liderazgo: Una nueva forma de dirigir, València, Universitat de València. SEGERMAN-PECK, Lily (1991): Networking and mentoring. A woman's guide, Londres, Judy PiatkusLdto. TUR AUSINA, Rosario (2014): «Los mitos de la participación equilibrada de mujeres y hombres en la toma de decisiones», en lgualdad y democracia: el género como categoría de análisis jurídico, València, Corts Valencianes, pp. 271-683.

V.AA. (2015): Mujeres y hombres en España, Madrid, INE.

WeBer, Max (1964): Economía y sociedad. Esbozo de sociología comprensiva, trad. J. Medina Echavarría, México, ed. J. Winckelmann, México, Fondo de Cultura Económica.

WEB TOD UMH: http://tod.edu.umh.es

WEB ONU MUJERES: http://www.unwomen.org/es/how-we-work/intergovernmental-support/worldconferences-on-women

WEB RADIO UMH: hHtp://radio.umh.es

WeB XARXA VIVES D'UNIVERSITATS: http://www.vives.org/serveis/liga-de-debat/liga-dedebat-universitari/

Recibido el 19 de marzo de 2017

Aceptado el 19 de mayo de 2017

BIBLID [1 139-1219 (2017) 22: 73-85] 